\begin{tabular}{l}
\multicolumn{5}{l}{ INTERNATIONAL BULLETIN OF BACTERIOLOGICAL } \\
Volume 5 \\
NOMENCLATURE AND TAXONOMY
\end{tabular}
MEMBERSHIP OF COMMITTEES, COMMISSIONS, AND SUBCOMMITTEES
1955

\author{
INTERNATIONAL COMMITTEE ON \\ BACTERIOLOGICAL NOMENCLATURE
}

Provision 4 of the International Bacteriological Code of Nomenclature established a permanent International Committee on Bacteriological Nomenclature "so constituted that wherever practicable each country is represented by at least one member and no country by more than five. Nominations for membership are made preferably by one or more of the microbiological societies in each country."

The Permanent Secretaries will be grateful for information relative to any inaccuracies.

\title{
Officers
}

Chairman: MURRAY, E.G.D. - Dept. of Bacteriology and Immunology, McGill University, Montreal, Canada.

Vice-Chairman: VARTIOVAARA, J. Unto - Université de Helsinki, Helsinki, Finland.

Permanent Secretary for Medical and Veterinary Bacteriology COWAN, Dr. S.T. - National Collection of Type Cultures, Central Public Health Laboratory, Colindale, Avenue, London N.W.9, England

Permanent Secretary for Nonmedical Bacteriology:

WIKEN, Prof. Torsten - Inst. f. Bakteriologie u. Gar ungsbiologie., Eidgenoss. Techn. Hochschule, Universitátstrasse 2, Zürich 6, Switzerland.

\section{Life Members}

ST. JOHN-BROOKS, Dr. Ralph - Department of Bacteriology, Trinity College, Dublin, Ireland.

BREED, Prof. R.S. - 6 Sunset Drive, Geneva, New York, U.S.A. 


\section{Members of Committee}

\section{ARGENTINA}

SORDELli, Prof. A. - Posodas 1553, Martinez F.C.N.G. B.N., Buenos Aires.

SORIANO, Prof. Ing. Santos, - Instituto de Microbiologia Agricola, Villa Orturar-Suc. 17, Buenos Aires.

\section{AUSTRALIA}

MUNCH-PETERSON, Dr. E. - Div. Animal Health and Nutrition, Flemington Road and Park Street, Parkville, N.2, Victoria.

RUBBO, Prof. S.D. - Department of Bacteriology, The University, Melbourne, Victoria.

SKERMAN, Prof. V.B.D. - Department of Bacteriology, Medical School, Queensland University, Herston Road, Brisbane, Queensland.

\section{AUSTRIA}

EISLER-TERRAMARE, Prof. Michael, - Staatl. Ser otherapeut. Institut, Universität, Vienna 10.

JANKE, Prof. Alexander - Technische Hochschule, Waldeckgasse 3, Wien XVIII 110.

SZYLVINI, Prof. Ing. - Institut fur angewandte Mikrobiologie, Hochschule far Bodenkultur, Michaelstr. 25, Vienna 18.

ZISCHKA, Dr. R.L. - Pathol. Anatomisches Institut.

Spitalgasse 4, Vienna.

\section{BELGIUM}

BRUYNOGHE, Prof. Richard.- Department of Bacteriology, University of Louvain.

RENAUX, Prof. Ernest.- Faculté de Médicine et de Pharmacie, Université Libre de Bruxelles, 115 Boulevard de Waterloo, Brussels.

\section{BRAZIL}

BITANCOURT, Dr. Agesilau.- Instituto Biologico, Caixa Postal 7119 Sæ̃o Paulo, Estado de Såo Paulo. 
BACTERIOLOGICAL NOMENCLATURE AND TAXONOMY

CRUZ, Dr. Oswaldo, filho.- Instituto Oswaldo Cruz, Caixa Postal 926, Rio de Janeiro, D.F.

DA ROCHA LAGOA, Dr. Francisco de Paulo.- Instituto Oswaldo Cruz, Caixa Postal 926, Rio de Janeiro, D.F. PENHA, Dr. Adolpho Martins,- Instituto Biologico, Caixa Postal 7119 Sào Paulo (Estado de São Paulo).

PENIDA, Dr. J.C.N. - Instituto Oswaldo Cruz, Caixa Postal 926, Rio de Janeiro D.F.

\section{BRITISH WEST INDIES}

THAYSEN, Dr. A.C. - Director, Colonial Microbiological Institute, P.O. B ox 584, Port-of-Spain, Trinidad

\section{BULGARIA}

MARKOFF, Prof. W. - Dept. of Bacteriology, University of Sofia, 3 Rue Dounau, Sofia.

PETROFF, Prof. T. - Department of Hygiene, University of Sofia, Sofia.

\section{CANADA}

LOCHHEAD, Dr. A.G. - Division of Bacteriology, Central Experimental Farm, Ottawa, Ontario.

MORIN, Prof. E.J. - Dept. of Bacteriology, Laval University, Quebec, P.Q.

MURRAY, Prof. E.G.D. - Dept. Bacteriology, McGill University, Montreal, P.Q.

REED, Prof. Guilford B. - Dept. Bacteriology, Medical School, Queens University, Kingston, Ontario.

VAN ROOYEN, Dr. C.E. - Connaught Laboratories, University of Toronto, Toronto 5, Ontario.

\section{CHI LE}

GRAF-MARIN, Dr. Alberto-Professor of Agricultural Microbiology, Universidad de Chile, Casilla 370 , Santiago.

SAN MIGUEL, Dr. Julio - Faculdad de Ciencias Pecuarias y Medicina Veterinaria, Universidad de Chile, Casilla 370 , Santiago. 
VACCARO, Dr. Hugo,- Instituto de Microbiologia e Imunologia, Casilla 370, Santiago.

WILHELM, Dr. Ottmat.- Professor, Universidad de Concepcion, Concepcion.

\section{CZECHOSLOVAKIA}

MALEK, Dr. Ivan. - Department of Bacteriology, University of Praha, Preslova 7, Praha.

RASKA, Dr. Karel-- State Institute of Public Health, Srobárova 48, Praha III.

\section{DENMARK}

BUCHWALD, Prof. Dr. N. Fabritius, - Kgl. Veterinaer og Landbohфjskole, Balowsvej, Copenhagen.

CHRISTIANSEN, Prof. M. - Serum Lab., Veterinary School, Balowsvej, Copenhagen.

KAUFFMANN, Dr. F. - Statens Seruminstitut, Amager Blvd. 80, Copenhagen $S$.

ØRSKOV, Dr. J. - Statens Seruminstitut, Amager Blvd. 80, Copenhagen $S$.

\section{EIRE}

GRIMES, Prof. Michael - Dairy Institute, University of Cork, Cork.

FINLAND

GYLLENBERG, Dr. H.H.G. - Messeniuksenk. 7 A 18, Helsinki.

MUSTAKALLIO, Prof. E. - Institute of Bacteriology, Turku. PENTTINEN, Dr. E. - Maurink. $4 \mathrm{~A}$, Helsinki.

VARTIOVAARA, Dr. Unto.- Dept. of Microbiology, The University, Helsinki.

\section{FRANCE}

HAUDUROY= Prof. Paul. - Directeur du Centre de Collection de Types Microbiens, 19 Ave. César Roux, Lausanne, Switzerland. 
BACTERIOLOGICAL NOMENCLATURE AND TAXONOMY

LEPINE, Dr. P. - Institut Pasteur, 25 Rue du Dr. Roux, Paris XVe.

LWOFF, Dr. A. - Institut Pasteur, 28 Rue du Dr. Roux, Paris XVe.

PREVOT, Dr. A.R. - Institut Pasteur, 25 Rue du Dr. Roux, Paris XVe.

SEGRETAIN, Dr. G. - Institut Pasteur, 25 Rue du Dr. Roux, Paris XVe.

\section{GERMANY}

HAUPT, Prof: Dr. Herbert.- Veterinärhygienisches Institut,

Frankfurterstrasse 87 , Giessen.

SEELEMANN, Prof. Dr. M. - Institut far Milchhygiene,

Hermann Weigmannstr 3/11 Kiel.

SEELIGER, Dr. H. - Hygiene-Institut, Venusberg, Bonn.

STAPP, Prof. Dr. C. - Direktor Biologische Zentralanstalt, 20b Braunschweig-Gliesmarode. Messeweg 25.

\section{GREAT BRITAIN AND NORTHERN IRELAND}

ANDREWES, Dr. C.H. - National Institute for Medical

Research, The Ridgeway, Mill Hill, London N.W. 7 . GIBSON, Dr. T. - College of Agriculture, 13 George Square, Edinburgh 8, Scotland.

MILES, Dr. A.A. - National Institute for Medical Research, The Ridgeway, Mill Hill, London N.W. 7 .

RAMSBOTTOM, Dr. J. - British Museum (Natural History)

Cromwell Road, London S.W. 7 .

WILSON, Dr. G.S. - Public Health Laboratory Service, 38 Old Queen St., Westminster, London S. W. 1 .

\section{HUNGAR Y}

MANNINGER, Prof. R. - Director, State Veterinary Hygienic Institute, Hungaria Korut 23-26, Budapest XIV.

\section{ISRAEL}

ASCHNER, Dr. M. - Department of Bacteriology, Hebrew University, Jerusalem. 


\section{ITALY}

ARNAUDI, Prof. Carlo. - Instituto di Microbiologia della

Facoltà di Agraria, Università di Milano, Via Celaria, Milan.

CASTELlani, Prof. Aldo.- Instituto de Medecina Tropical, Travessa do Franco, Lisboa, Portugal

CIFERRI, Prof. R. - Instituto Botanico, Università di Pavia, Via Scopoli, P.O. Box 165, Pavia.

DE ROSSI, Prof. Gino, - Via Buccari 3, Rome.

PENSO, Dr. G. - Instituto Superiore di Sanità, Viale Regina Margherita 299, Rome.

\section{JAPAN}

ASAI, Prof. Dr. Toshinobu, - Faculty of Agriculture, Univ. of Tokyo, Mukogaoka-Yayoimachi, Bunkyo-ku, Tokyo.

KOJIMA, Dr. Sabura.- Director of the National Institute of Health 1, Shiba-Shirokane-Daimachi, Minato-ku, Tokyo.

KOMINAMI, Dr. Kiyoshi. - Nagao Research Institute, 387, 6, Cho-me, Kitashinagawa, Shinagawa-ku, Tokyo.

\section{NETHERLANDS}

KLUYVER, Prof. Dr. A.J. - Technical High School, Nieuwe Laan 3, Delft.

RUYS, Prof. A.Charlotte. - Laboratory of Hygiene of Univ. of Amsterdam, Mauritskade 57, Amsterdam.

SMIT, Prof. Dr. Jan.- Universite Agronomique, Wageningen. VERLINDE, Prof. Dr. J.D. - Université de Leyden, Nederland Institut voor Praeventive Geneeskunde, Wassenaarschweg 56, Leyden.

WOLFF, Prof. Dr. J.W. - Konikluk Institut voor de Tropen, Amsterdam.

\section{NEW ZEALAND}

BUDDLE, M.B., (B.V.Sc.) - Wallaceville Animal Research Station, Private Bag, Wellington.

KIRSCHNER, Dr. L. - Medical School, University of Otago, Dunedin, $\mathrm{Cl}$.

WHITEHEAD, Dr. H.R. - Dairy Research Institute, Massey College, Palmerston North. 
BACTERIOLOGICAL NOMENCLATURE AND TAXONOMY

\section{NOR W AY}

HENRIKSEN, Dr. S. Dick - Statens Institutt for Folkehelse, Geitmyrsvegen 75, Oslo.

THJ TTA, Prof. Th. - Bacteriological Institute, University of Oslo, Oslo.

VOSS, Prof. J. - State Institutt for Folkehelse, Geitmeyrs vegen 75 , Oslo.

\section{POLAND}

LEGEZYNSKI, Prof. St. - Department of Bacteriology,

State Institute of Hygiene, Chocimska 28, Warsaw. PRZESMYCKI, Prof. F. - State Institute of Hygiene,

Chocimska 28, Warsaw.

SZYMANOSKI, Prof. Dr. F. - Dept. of Bacteriology, The

University, Lodz.

\section{PORTUGAL}

OLIVEIRA, Prof. Candido de. - Instituto Bacteriol6gico

Camara Pestana, Rua do Instituto Bacteriologico, Lisbon.

\section{RUMANIA}

IONESCU-MIHAIESTI, Prof. Dr. C. - Faculty of Medicine, Splaiul Dr. Carol Davilla 6, Bucharest VI. ZOTTA, Prof. Dr. G. - Splaiul Dr. Carol Davilla 6, Bucharest VI.

\section{SPAIN}

FALCO, Dr. A. Ruiz.- Institutp Nacional de Hygiene, Madrid.

\section{SWEDEN}

GARD, Prof. Sven.- State Bacteriological Laboratory, Karolinska Institutet, Box 764, Stockholm 1 . HÜLPHERS, Prof. Gustav, - Dept. Bacteriology, Veterinary College, Stockholm. NILSSON, Prof. R. - Kungl. Lantbrukshogskölan, Uppsala. 


\section{SWITZER LAND}

FREI, Prof. W. - Veterinăr-Pathologisches Institut, University of Zürich, Zürich.

GRASSET, Prof. Dr. E. - Institut d'Hygiene et de Bacteriologie de 1'Université de Geneve. Geneve.

KÄSTLI, Prof. Dr. P. - Eidg. Milchwirtschaftliche u. Bakteriologische Versuchsanstalt, Liebefeld-Bern. MOOSER, Prof. Dr. H. - Hygienisches Institut der Universität Zürich, Gloriastrasse 32, Zürich.

TERRIER, Dr. Ch. - Stations federales d'essais viticoles, Arboricoles et de chimie agricole, Montagibert Lausanne.

\section{UNITED STATES OF AMERICA}

ALLEN, Dr. Oscar N. - Department of Bacteriology, Univ. of Wisconsin, Madis on 6, Wisconsin.

BROWN, Dr. J. Huward - 1640 Gorsuch Ave., Baltimore 18, Maryland.

BUCHANAN, Prof. R.E. - Room 316 Curtiss Hall, Iowa State College, Ames, Iowa.

VAN NIEL, Prof. C.B. - Hopkins Marine Station, Pacific Grove, California.

\section{URUGUAY}

HOR MAECHE, Prof. E. - Instituto de Higiene, Faculdad de Medicina, Av. A. Ricaldoni, 3051, Montevideo.

$$
\text { U.S.S.R. }
$$

HOROWITZ-WLASSOWA, Prof. - 1 Linia, 54, App. 3, Leningrad 53 , W.O.

ISSATCHENKO, Prof. Dr. B. - Institute of Experimental Medicine, 96 Baltiiskii poselok 13, Moscow 96. 
BACTERIOLOGICAL NOMENCLATURE AND TAXONOMY

\section{JUDICIAL COMMISSION OF THE} INTERNATIONAL COMMITTEE

Provision 5 of the International Bacteriological Code of Nomenclature established a Judicial Commission to consist of fourteen members, twelve elected by the members of the International Committee on Bacteriological Nomenclature from its membership, together with the two Permanent Secretaries. The Commissioners are elected to serve in three classes of four Commissioners each, one class retiring at the close of the meeting of each Congress of the International Association of Microbiologists.

Class of 1956 (Term expiring at the close of the seventh Congress).

LWOFF, Prof. A. - Institut Pasteur, 28 Rue du Dr. Roux, Paris XVe, France.

PENIDO, Prof. J.C. - Instituto Oswaldo Cruz, Caixa Postal 926, Rio de Janeiro, D.F., Brazil.

RENAUX, Prof. E. - Faculté de Medicine et de Pharmacie, Université Libre de Bruxelles, 115 Blvd. de Waterloo, Brussels, Belgium.

SORDELLI, Prof. A. - Posodas 1553, Martinez F.C.N.G.

B.N., Buenos Aires, Argentina.

Class of 1959 (Term expiring at the close of the eighth Congress).

ANDREWES, Dr. C.H. - National Institute for Medical Research, The Ridgeway, Mill Hill, London NW 7, England.

RAMSBOTTOM, Dr. J. - British Museum (Natural History) Cromwell Road, London SW 7, England.

THJ $\varnothing$ T TA, Prof. Th. - Bacteriological Institute, University of Oslo, Oslo, Norway.

VAN NIEL, Prof. C.B. - Hopkins Marine Station, Pacific Grove, California, U.S.A.

Class of 1962 (Term expiring at the close of the ninth Congress).

BUCHANAN, Prof. R.E. (Chairman) - 316 Curtiss Hall, Iowa State College, Ames, Iowa, U.S.A.

KLUYVER, Prof. A.J. - Technical High School, Nieuwe Laan 3, Delft, Netherlands. 
MILES, Prof. A.A. - National Institute for Medical Research, The Ridgeway, Mill Hill, London N.W. 7, England.

MURRAY, Prof. E.G.D. - Department of Bacteriology and Immunology, McGill University, Montreal, Canada.

Permanent Joint Secretaries

COWAN, Dr. S.T. - National Collection of Type Cultures, Central Public Health Laboratory, Colindale Avenue, London NW 9, England.

WIKEN, Prof. Torsten. - Inst. f. Bakteriologie u. Gärungs biologie., Eidgenoss, Techn. Hochschule, Universitaltstrasse 2, Zurich 6, Switzerland.

TAXONOMIC SUBCOMMITTEES

OF THE INTERNATIONAL COMMITTEE

Provision 4 of the International Bacteriological Code of Nomenclature authorized the appointment of subcommittees of experts to consider and report on the classification of special groups of microorganisms and on other phases of the taxonomy. Eight such taxonomic subcommittees have been appointed. In some cases, the subcommittees have designated groups of experts to study andreport uponcertain subdivisions of the taxa under consideration.

\section{Subcommittee on Clostridium}

COWAN, Dr. S. T. (Ex-officio) - See address above.

FREDETTE, Dr. V. - 3170 Maplewood, Montreal, Canada MC CLUNG, Dr. L.S. - Dept. of Bacteriology, Indiana University, Bloomington, Indiana, U.S.A.

MC COY, Miss E - Dept. of Agricultural Bacteriology, University of Wisconsin, Madison, Wisconsin, U.S.A. PRÉVOT, Dr. A.R. (Chairman) - Institut Pasteur, 25 Rue du Dr. Roux, Paris XVe, France.

ROSS, Miss H.E. - National Collection of Type Cultures, Central Public Health Laboratory, Colindale Avenue, London NW 9, England.

SPRAY, Dr. R.S. - Box 742, Cumberland, Wisconsin, U.S.A. THAYSEN, Dr. A.C. - Colonial Microbiological Research

Institute, Box 581, Port of Spain, Trinidad, B.W.I. 
BACTERIOLOGICAL NOMENCLATURE AND TAXONOM Y

TURNER, Dr. A.W. - CSIR Animal Health Laboratory, Parkville N2, Victoria, Australia.

Subcommittee on Enterobacteriaceae

HORMAECHE, Prof. E. (Chairman) - Instituto de Higiene, Av. A. Ricaldoni 3051 , Montevideo, Uruguay.

BENSTED, Lt. Col. H.J. - Central Public Health Laboratory, Colindale Avenue, London NW 9, England

BOYD, Brig. J.S.K. - The Wellcome Laboratories of Tropical Medicine, 183-193 Euston Rd., London NW 1, England.

BRUNER, Dr. D.W. - Dept. of Pathology and Bacteriology, New York State Veterinary College, Cornell University, Ithaca, New York, U.S.A.

CARLQUIST, Lt. Col. P.R. - 4 th Army Area Medical Laboratory, Ft. Sam Houston, Texas, U.S.A.

COWAN, Dr. S.T. (Ex-officio) - Central Public Health Laboratory, Colindale Ave., London NW 9, England.

EDWARDS, Dr. P.R. - U.S. Public Health Service, Communicable Disease Center, Enteric Bacteriology Laboratories, Chamblee, Georgia, U.S.A.

FERGUSON, Dr. W. W. - Michigan State Department of Health, Lansing 4, Michigan, U.S.A.

GIOVANARDI, Prof. Dr. A. - Instituto d'Igiene, Via Fr. Sforza 35, Milano, Italia.

KAUFFMANN, Dr. F. - Statens Seruminstitut, Copenhagen

- S, Denmark.

LeMINOR, Dr. L. - Chief, Laboratory Service, Institute Pasteur, 25 Rue du Dr. Roux, Paris, France.

OLIN, Prof. Dr. G. - Statens Bakteriologiska Laboratorium B ox 764, Stockholm 1, Sweden.

RAUSS, Prof. Dr. K. - The Hygienic Institute, Pecs, Hungary .

SEELIGER, Dr. H. - Hygiene-Institut, Bonn University, Bonn, Germany.

STUART, Prof. Dr. C.A. - Department of Bacteriology, Brown University, Providence, Rhode Island, U.S.A.

TAYLOR, Dr. Joan - Central Public Health Laboratory, Colindale Avenue, London NW 9, England

EWING, Dr. W.H. (Secretary) - Communicable Disease Center, Enteric Bacteriology Laboratory, U.S. Public Health Service, Chamblee, Georgia, U.S.A. 


\section{Subcommittee on Leptospira}

Working Party

BORG PETERSEN, Dr. C. - Statens Seruminstitut, Copenhagen, Denmark.

BROOM, Dr. J.C. (Secretary) - Wellcome Laboratories,

183-193 Euston Road, London NW 1, England.

MEYER, Dr. K.F. - Medical Center, University of California, San Francisco, California, U.S.A.

WIESMANN, Dr. W. - Kanton Hospital, St. Gallen, Switzerland.

WOLFF, Prof. J.W. (Chairman) - Institute of Tropical Medicine, Maurits Kade 57, Amsterdam, Holland.

Other Members

BABUDIERI, Prof. B. - Institute di Sanitá Superiore, Rome, Italy.

BALL, Dr. M. - University of California, Los Angeles 24, California, U.S.A.

BESSEMANS, Prof. Dr. A. - Institute of Hygiene, The University, Ghent, Belgium.

COWAN, Dr. S.T. (Ex-officio) - National Collection of Type Cultures, Central Public Health Laboratory, Colindale Avenue, London NW 9, England.

DAVIS, Dr. Gordon,- Rocky Mt. Laboratory, U.S. Public

Health Service, Hamilton, Montana, U.S.A.

de AZEVEDO, Prof. Fraga.- Instituto de Medicina Tropical, Lisbon, Portugal.

DERRICK, Dr: E.H. - Queensland Institute of Medical Research, William Street, Brisbane, Australia.

de SALLES GOMES, Dr. L. - Instituto Adolpho Lutz, Av.

Dr. Arnaldo, São Paulo, Brazil.

GUIDA, Dr. V.O. - Instituto Biologica de São Paulo, São Paulo, Brazil.

KOLOCHINE-ERBER, Mad. B. - Institut Pasteur, Paris, France.

MUNIZ, Dr, Julio, Institut Oswaldo Cruz, Caixa Postal 926, Rio de Janeiro, D.F. Brazil.

RENNELLA, Prof. E. - Bacteriological Institute Malbran Buenos Aires, Argentina.

SANTOS, Dr. Maria - Institut Oswaldo Cruz, Caixa Postal 926, Rio de Janeiro, D.F. Brazil. 
BACTERIOLOGICAL NOMENCLATURE AND TAXONOMY

SCHLOSSBERGER, Dr. H. - Hygienisches Institut der Stadt und Universităt, Paul Ehrlich Strasse 40, Frankfurt a/M. Süd 10, Germany

van der HOEDEN, Dr. J. - Director of the Veterinary Institute, Tel Aviv, Israel.

VAN RIEL, Prof. J. - Institute of Tropical Medicine, Antwerp, Belgium.

van THIEL, Prof. J. - Institute of Tropical Medicine, Rápenburg 33, Leiden, Holland.

WILHELM, Prof. Ohmat.- University of Concepcion, Concepcion, Chile.

Subcommittee on Medical and Veterinary Mycopathology

AINSWORTH, Dr. G.C. - Department of Botany, Universtty College, Exeter, Devon, Great Britain.

de ALMEIDA, Dr. Floriano - Seccão Micalogia, Departamento de Microbiologia, Faculdade de Medicina, Universidade de Sł̊o Paulo, Sł̃o Paulo, Brazil.

CARRION, Dr. A.L. - San Juan, Puerto Rico.

CIFERRI, Prof. R. - Instituto Botanico, Pavia Università, Via Scopoli, Pavia, Italy.

COWAN, Dr. S.T. (Ex-officio) - National Collection of Type Cultures, Central Public Health Laboratory, Colindale Avenue, London NW 9, England.

de AREA LEAO, Dr. A.E. - Laboratorio de Micologia, Institut Oswaldo Cruz, Caixa Postal 926, Rio de Janeiro, Brazil.

DUNCAN, Dr. J.T. - 31 Nightingale Road, Rickmansworth, Herts, England.

EMMONS, Dr. C.W. - National Institute of Health, U.S. Public Health Service, Bethesda 4, Maryland, U.S.A.

MACKINNON, Prof. Juan E. -. Instituto de Higiene, Facultad de Medicina, Av. Ramon Anador 3051, Montevideo, Uruguay.

NEGRONI, Dr. P. - Pichincha 830, Buenos Aires, Brazil. NICKERSON, Dr. W.J. (Secretary) - Microbiological Institute, Rutgers University, New Brunswick, New Jersey, U.S.A.

REDAELLI, Prof. P. - Instituto Anatomia Patologica, Università Via F. Sforza 38, Milan, Italy. 
S KINNER, Dr. C.E. - Washington State College, Pullman, Washington. U.S.A.

WESTERDIJK, Prof. Johanna, Centralbureau voor Schimmelcultures, Javalaan 4, Baarn, Holland.

Subcommittee on Mycobacterium

CHAIN, Dr. E.B.- Institut Superior de la Sante, Rome, Italy .

DUBOS, Dr. R.J. - Rockefeller Institute, 66th and York Avenue, New York 21, New York, U.S. A.

GORDON, Dr. Ruth E. - Microbiological Institute, Rutgers University, New Brunswich, New Jersey, U.S.A.

HAGAN, Dr. W.A. - New York State Veterinary College, Ithaca, New York, U.S.A.

HANKS, Dr. John - Leonard Wood Memorial Leprosy Foundation, Harvard Medical School, Boston, Massachusetts, U.S.A.

HAUDUROY, Prof. P. (Chairman) - 19 Avenue de CésarRoux, Lausanne, Switzerland.

JENSEN, Prof. K.A. - Universitats-Institut for Almindelig, Pathologie Juliane Maries Veg 22, Copenhagen, Denmark.

PENSO, Dr. G. (Secretary) - Instituto Superiore de Sanita, Viale Regina Margherita, 299, Rome, Italy.

REED, Prof. G.D. - Dept. of Bacteriology, Medical School, Kingston, Canada.

WELLS, Dr. A.Q. - Sir William Dunn School of Pathology, South Parks Road, Oxford, England.

WIKEN, Prof. Torsten (Ex-officio) - Inst. f. Bakteriologie u. Gárungsbiologie, Eidgenoss. Techn. Hochschule, Universitătstrasse 2, Zürich 6, Switzerland.

YAMAZAKI, Dr. Izue - Faculty of Agriculture, Kyushu University, Hakozaki-cho, Fukuoka-shi, Japan.

Subcommittee on Neisseriaceae

BRANHAM, Dr. Sara E. (Secretary) - National Institute of Health, Bethesda, Maryland, U.S.A.

BENSTED, Lt. Col. H.J. - Central Public Health Laboratory Colindale Avenue, London NW 9, England.

COHEN, Miss Sophia M. - New York State Dept. of Health, New Scotland Avenue, Albany 1, New York, U.S.A. 
BACTERIOLOGICAL NOMENCLATURE AND TAXONOMY

COWAN, Dr. S.T. (Ex-officio) - National Collection of Type cultures, Central Public Health Laboratory, Colindale MILLER, Dr. C. Philip - Dept. of Medicine, University of Chicago, Chicago 37, Illinois, U.S.A.

MURRAY, Prof. E.G.D. (Chairman) - Bacteriology Dept. McGill University, Montreal, Canada.

PELCZAR, Dr. M.J. - University of Maryland, College Park, Maryland, U.S.A.

PRÉVOT, Dr. A.R. - Institut Pasteur, 25 Rue du Dr. Roux, Paris XVe, France.

ST. JOHN-BROOKS, Dr. Ralph - Department of Bacteriology, Trinity College, Dublin, Ireland.

THJØТTA, Prof. Th. - Bacteriological Institute, University of Oslo, Oslo, Norway.

Subcommittee on Phage Typing of Staphylococcus

WILLIAMS, Dr.R.E.O. (Convenor) - Streptococcus Reference Laboratory, Colindale Avenue, London, NW 9, England.

COWAN, Dr. S.T. (Ex-officio) - See address above.

The complete list of members of this subcommittee had not been received at time of publication of this Bulletin. It will appear in the next is sue.

Subcommittee on Streptococcus and Pneumococcus

ALLISON, Dr. V.D. (Chairman) - North Ireland Hospitals Authority, 51 Lisburn Road, Belfast, Northern Ireland. EDDY, Dr. B. - National Institute of Health, Bethes da 14, Maryland, U.S.A.

FERRIS, Dr. A.A. - Fairfield Hospital, Melbourne, N 20, Australia.

FRY, Dr. R.M. - Public Health Laboratory Service, Tennis Court Road, Cambridge.

HANSEN, Dr. P.A. - University of Maryland, College Park, Maryland, U.S.A.

KAUFFMANN, Dr. F. - State Serum Institute, Amager

Boulevard 80, Copenhagen, Denmark.

LANCEFIELD, Dr. R. - Rockefeller Institute for Medical

Research, 66th St. and York Avenue, New York 21,

New York, U.S.A. 
LUND, Dr. E.M. - State Serum Institute, Amager Boulevard 80 , Copenhagen, Denmark.

MATTICK, Dr. A.T.R. - National Institute for Research in Dairying, Shinfield, Reading, Berks, England.

MAXTED, Mr. W.R. - Streptococcus Reference Laboratory, Colindale Avenue, London, NW 9, England.

MOCQUOT, Dr. G. - Station Centrale de Microbiologie et Recherches Laitieres, Jouy-en-Josas, France.

NIVEN, Dr. C.F. Jr. - American Meat Institute Foundation, 939 E. 57th Street, Chicago, Illinois, U.S.A.

PAKULA, Dr. R. - State Institute of Hygiene, Ul. Chocimska 24, Warsaw, Poland.

PAT TISON, Mr. I.H. - Agricultural Research Council, Field Station, Compton, Newbury, Berks, England.

SEELEMANN, Prof. M. - Institut für Milchhygiene, Hermann Weigmannstr. 3/11, Kiel, Germany.

SHERMAN, Dr. J.M. - Dept. of Bacteriology, New York

State College of Agriculture, Cornell University, Ithaca, New York, U.S.A.

SKADHAUGE, Dr. K. - State Serum Institute, Amager Boulevard 80, Copenhagen, Denmark.

STABLEFORTH, Dr. A.W. - Ministry of Agriculture and Fisheries, New Haw, Weybridge, Surrey, England.

UPDYKE, Dr. E. - Streptococcus Laboratory, P.O. Box 185, Chamblee Georgia, U.S.A.

WAHL, Dr. R. - Institut Pasteur, 25 Rue du Docteur Roux, Paris XV, France.

WILLIAMS, Dr. R.E.O. (Secretary) - Streptococcus Reference Laboratory, Colindale Avenue, London NW9, England.

WIKEN, Prof. Torsten (Ex-officio) - Inst. f. Bakteriologie u. Gárungsbiologie, Eidgenoss, Techn. Hochschule, Universitätstrasse 2, Zürich 6, Switzerland.

WILSON, Dr. A.T. - Alfred I. du Pont Institute of the Nemours Foundation, Wilmington 99, Delaware, U.S.A.

Subcommittee on Viruses

\section{Animal Viruses}

ANDREWES, Dr.C.H. (Chairman) - National Institute for Medical Research, The Ridgeway, Mill Hill, London NW 7, England. 
BACTERIOLOGICAL NOMENCLATURE AND TAXONOMY

BERGOLD, Dr. G.H. - Laboratory of Insect Pathology, P.O. Box 490, Sault Ste. Marie, Ontario, Canada. BURNET, Sir Macfarlane, F.R.S. - Walter and Eliza Hall Institute, Royal Melbourne Hospital, Parkville N2, Melbourne, Australia.

GARD, Dr. Sven, Dept. of Virus Research, Karolinska Institutet, Box 764, Stockholm 1, Sweden.

HAMMON, Dr. W. McD. - Dept. of Epidemiology, Graduate School of Public Health, University of Pittsburgh, Pittsburgh 13, Pennsylvania, U.S.A.

HENNEBERG, Prof. G. - Robert Koch Institut für Hygiene, Fohrerstrasse 2, Berlin N65, Germany.

LEPINE, Dr. P. - Institut Pasteur, 25 Rue du Docteur Roux, Paris XVe, France.

MAGRASSE, Instituto di Patologia Medica, Universita di Napoli, Piazza L. Miraglia, Napoli, Italy.

ORSKOV, Dr. J. - Statens Seruminstitut, Amager Boulevard 80 , Copenhagen, Denmark.

RAKE, Dr. G. - E.R. Squibb and Sons, Squibb Building, 745 Fifth Avenue, New York 22, New York, U.S.A.

Arthropod-borne Encephalitis Virus Group.

HAMMON, Dr. W. McD. - See address above.

LEPINE, Dr. P. - See address above.

OLITSKY, Dr. P.K. - Rockefeller Institute for Medical

Research, 66th St. and York Avenue, New York 21

New York, U.S.A.

Herpes Group

ANDREWES, Dr. C.H. - See address above.

SABIN, Dr. A.B. - The Children's Hospital Research Foundation, University of Cincinnati College of Medicine, Elland and Bethesda Avenues, Cincinnati 29, Ohio, U.S.A.

SCOTT, Dr. T.F. McN. - Children's Hospital, 1740 Bainbridge Street, Philadelphia 47, Pennsylvania, U.S.A.

RIVERS, Dr. T.M. - Hospital of Rockefeller Institute, 66th St. and York Avenue, New York 21, New York, U.S.A.

Influenza Group

ANDREWES, Dr. C.H. - See address above.

BURNET, Sir Macfarlane.- See address above.

BANG, Dr. F.B. - The Johns Hopkins Hospital, Baltimore 5, Maryland, U.S.A. 
Insect-pathogenic Virus Group

BERGOLD, Dr. G.H. - See address above.

BIRD, Dr. F.T. - c/o Dr.G.H. Bergold.

HUGHES, Dr. K.M. - c/o Dr. G.H. Bergold.

STEINHAUS, Dr. E.A. - Laboratory of Insect Pathology,

Division of Biological Control, College of Agriculture,

Berkeley, California, U.S.A.

WYATT, Dr. G.R. - Laboratory of In sect Pathology, P.O.

Box 490, Sault Ste. Marie, Ontario, Canada.

Poliomyelitis Group

GEAR, Dr. J.H.S. - South African Institute for Medical

Research, Hospital Street, Johannesburg, South Africa

PAUL, Dr. J.R. - Section of Preventive Medicina, Yale

University School of Medicine, 333 Cedar Street,

New Haven 11, Connecticut, U.S.A.

von MAGNUS, Dr. H. - Statens Seruminstitut, Amager

Boulevaid 80, Copenhagen, Denmark.

Pox Group

BURNET, Sir Macfarlane.- See address above.

GOODPASTURE, Dr. E.W. - Department of Pathology,

Vanderbilt University, Nashville, Tennessee, U.S.A.

HORGAN, Dr. E.S. - Virus Research Institute, P.O. Box

49, Entebbe, Uganda, East Africa.

Psittacosis-lymphogranuloma Group.

BEDSON, Prof.S.P., F.R.S. - The Middlesex Hospital,

London W 1, England.

GONNERT, Prof. R. - Chemotherapeutisches Laboratorium der Farbenfabriken Bayer, Wuppertal-Elberfeld,

Germany.

RAKE, Dr. G. - See address above.

\section{Bacterial Viruses}

ADAMS, Dr. M.H. - Dept. of Microbiology, University of New York, New York, N.Y. U.S.A.

CRAIGIE, Dr. J.,-O.B.E., F.R.S. - Imperial Cancer Re-

Search Fund Laboratories, Buttonhole Lane, Mill

Hill, London, NW 7, England.

NICOLLE, Dr. P. - Institut Pasteur, 25 Rue du Docteur

Roux, Paris XVe, France. 
BACTERIOLOGICAL NOMENCLATURE AND TAXONOMY

\section{Plant Viruses}

BAWDEN, Dr. F.C., F.R.S. - Dept. of Plant Pathology, Rothamsted Experiment Station, Harpenden, Herts. England.

BENNETT, Dr. C.W. - Sugar Plant Investigations, U.S. Dept. of Agriculture, P.O. Box 31, Riverside, California, U.S.A.

BITANCOURT, Dr. A. - Instituto Biologico, Caixa Postal 119-A, Sào Paulo, Brazil.

KOHLER, Dr. E. - Institut für Virusforschung, Dornbergstrasse 25/27, Celle, Germany.

LIMASSET, Dr. P. - Station Centrale de Path. Veg., Routede-St. Cyr, Versailles, France.

MC WHORTER, Dr. F.P. - Botany and Plant Pathology Dept., Oregon State College, Corvallis, Oregon, USA. PRENTICE, Dr. I. W. - Plant Pathology Laboratory, Ministry of Agriculture and Fisheries, Milton Road, Harpenden, Herts., England.

THUNG, Prof. T.H. - Institut voor Plantenziektenkundig Onderzoek, Binnenhaven $4 \mathrm{a}$, Wageningen, Holland. van SLOGERTEREN, Prof. E. - Laboratorium voor Bloembollen-Underzoek, Lisse, Holland.

COMMITTEE ON BACTERIA OF THE BOTANICAL CONGRESS

The International Botanical Congress has authorized the constitution of a Committee on Bacteria to serve as a liaison between bacteriologists and botanists.

ALLEN, Prof. O.N. (Chairman) - Dept. of Bacteriology, University of Wisconsin, Madison, Wisconsin, U.S.A. AINSWORTH, Dr. G.C. - University College, Exeter, Devon, England.

BREED, Prof. R.S. - 6 Subset Drive, Geneva, New York, U.S.A.

BUCHANAN, Prof. R.E. - Room 316 Curtiss Hall, Iowa State College, Ames, Iowa, U.S.A.

COWAN, Dr. S.T. - National Collection of Type Cultures, Central Public Health Laboratories, Colindale Avenue, London NW 9, England. 
LANJOUW, Prof. J. (Ex officio) - Botanisch Museum and Herbarium, Lange Nieuws traat 106, Utrecht, Holland. RAMSBOT TOM, Prof. John.- British Museum (Natural History), Cromwell Road, London SW 7, England. SERMOLLI, Prof. R.E.G. Pichi.- Herbarium Universitatis Florentinae, Instituto Botanico dell "Universita, Via Lamarmora No. 4, Firenze, Italy.

THJØTtA, Prof. Th. - Villaveien 30, V. Aker. pr., Oslo, Norway.

WIKÉN, Prof. T. - Inst. f. Bakteriologie u. Gärungsbiologie, Eidg. Techn. Hochschule, Universitătstrasse 2, Zürich, Switzerland. 